\title{
Content Based Image Retrieval from Auto Encoders Using Keras and Tensor Flow Python API a Deep Learning Technique
}

\author{
Vinitha .V1, Velantina.V2 \\ Post Graduates, University VTU, Belgaum
}

\begin{abstract}
As the technology is evolving new methods and techniques are determined and implemented in a smart way to improve and achieve a greater insight in this smart era. The retrieval of image is popularly growing in this emerging trend. In this paper we have used how to build a very simple image retrieval system using a special type of Neural Network called auto encoders. Here the images can be retrieved with visual contents textures, shape and this method of image retrieval is called content based image retrieval.
\end{abstract}

Keywords:- Image Retrieval, Keras, Tensor Flow, Neural Network.

\section{INTRODUCTION}

Content based means the search analyzes the contents of the image rather than the metadata such as keywords, tags, or descriptions associated with the image.

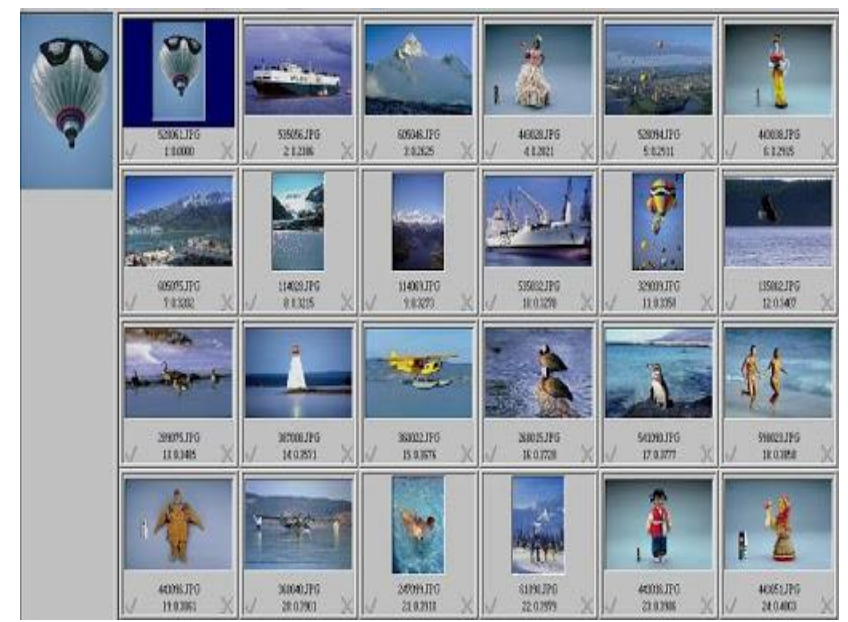

Fig 1:- Overview of image retrieval using content based technique among various images.
The comparison of two images in content-based image retrieval is using an image distance measure. The image distance measure compares the similarity of two images in various dimensions such as color, texture, shape, and others and the shapes is been identified by applying edge detection to an image.

\section{Deep learning}

Deep learning means specific subfield of machine learning which is new way of learning representations from data that puts an emphasis on model .

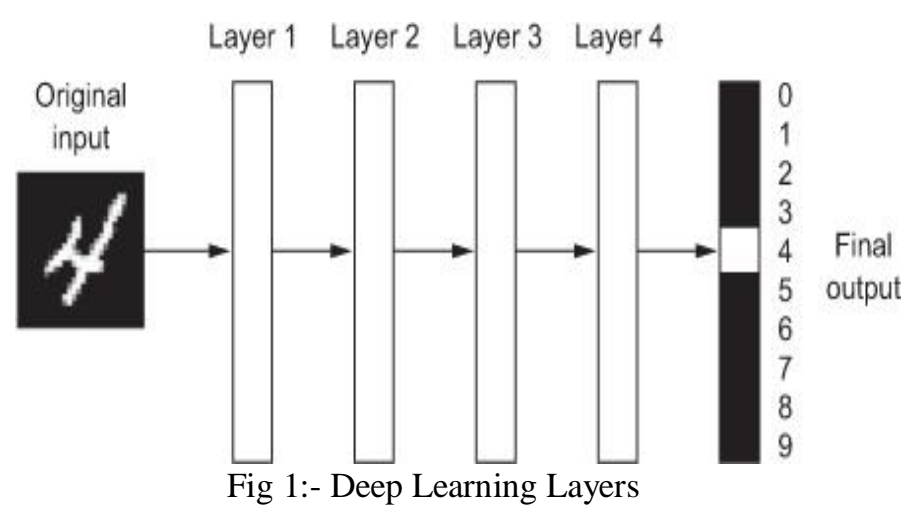

\section{PROPOSED SYSTEM}

The Auto encoders has two parts

Encoder: The network that consists of input into a latent space representation.

Decoder: The image search engines are similar to text search engines instead of presenting the search engine with a text query, we can provide an image query to the image search engine that finds all visually similar relevant images and train the auto encoder. 


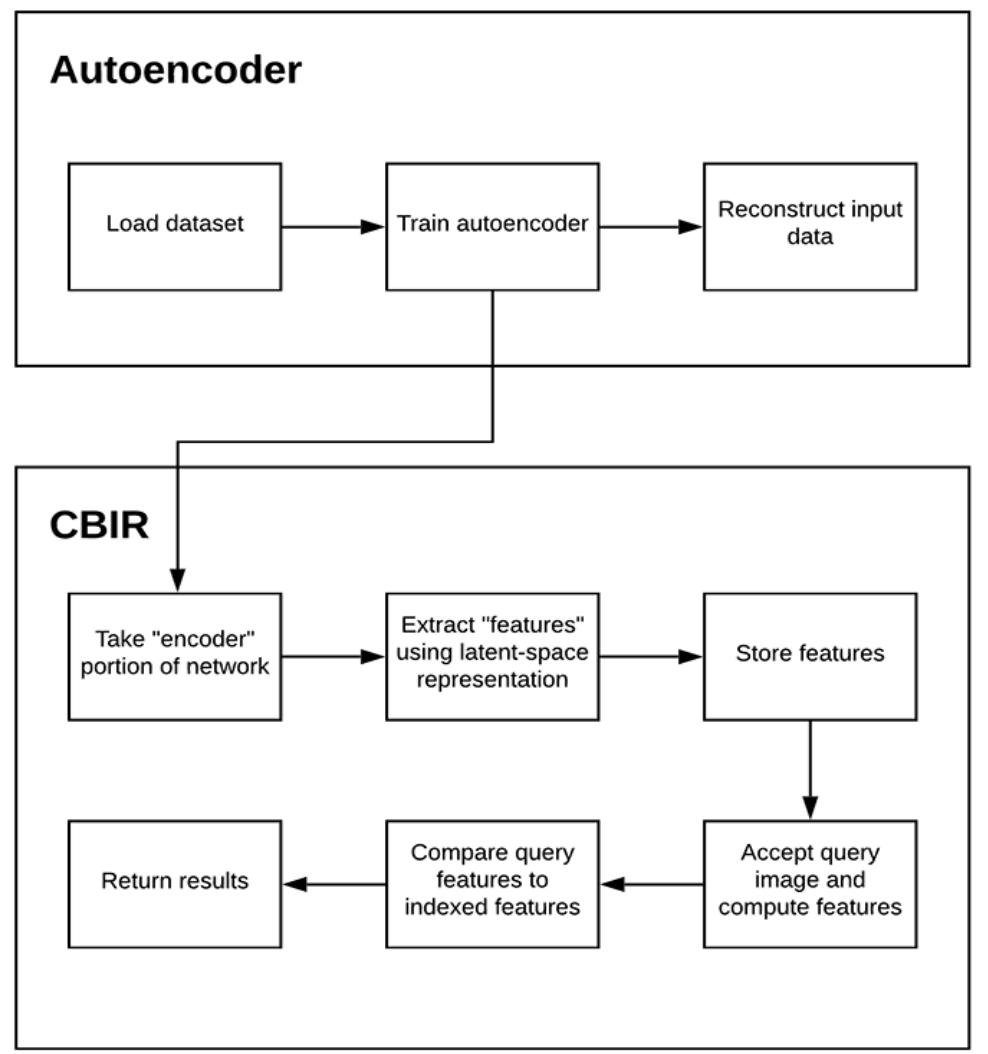

Fig 3:- Structure of Content Based Image Retrieval

Train an auto encoder on the input dataset in an unsupervised method and we use the auto encoder to extract and store features in an index and then search the index. The image retrieval system is build with an auto encoder that gives importance to latent space representation vector and the creation of an auto encoder training script is done using Keras and Tensor Flow.

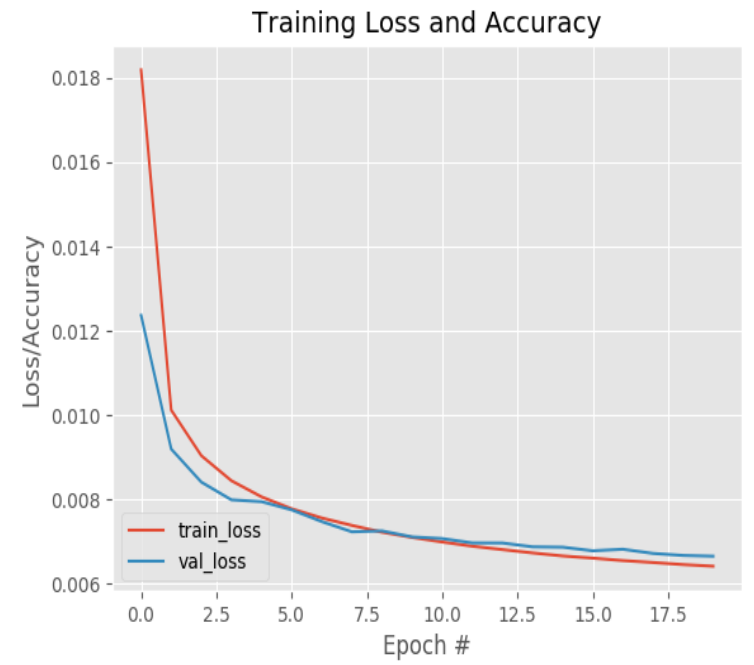

Fig 4:- Training an auto encoder with Keras and Tensor Flow for CBIR

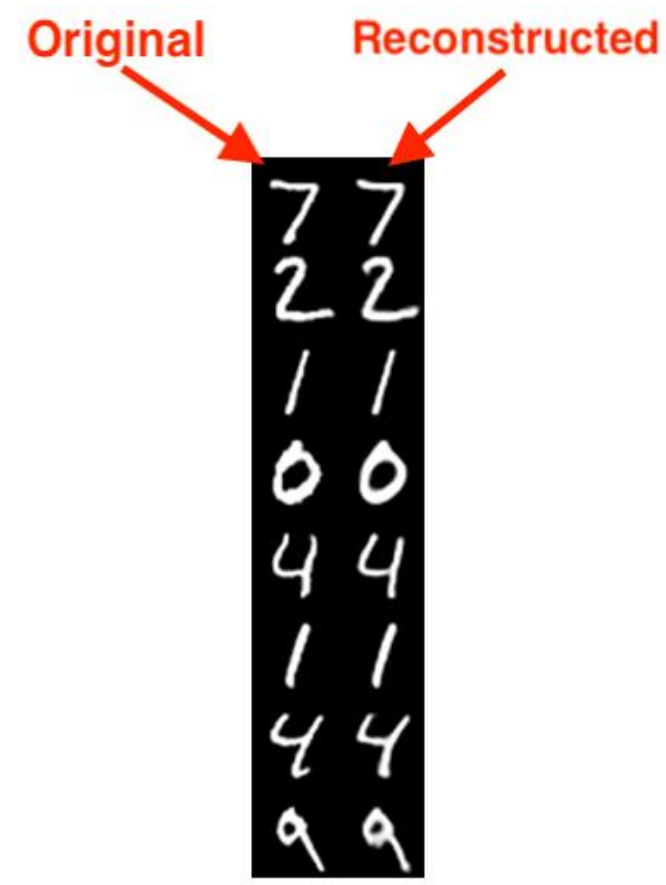

Fig 5:- The reconstructed data visualized through the auto encoder.

The example showing the image querying containing the digit 9 with auto encoder image retrieval system. 


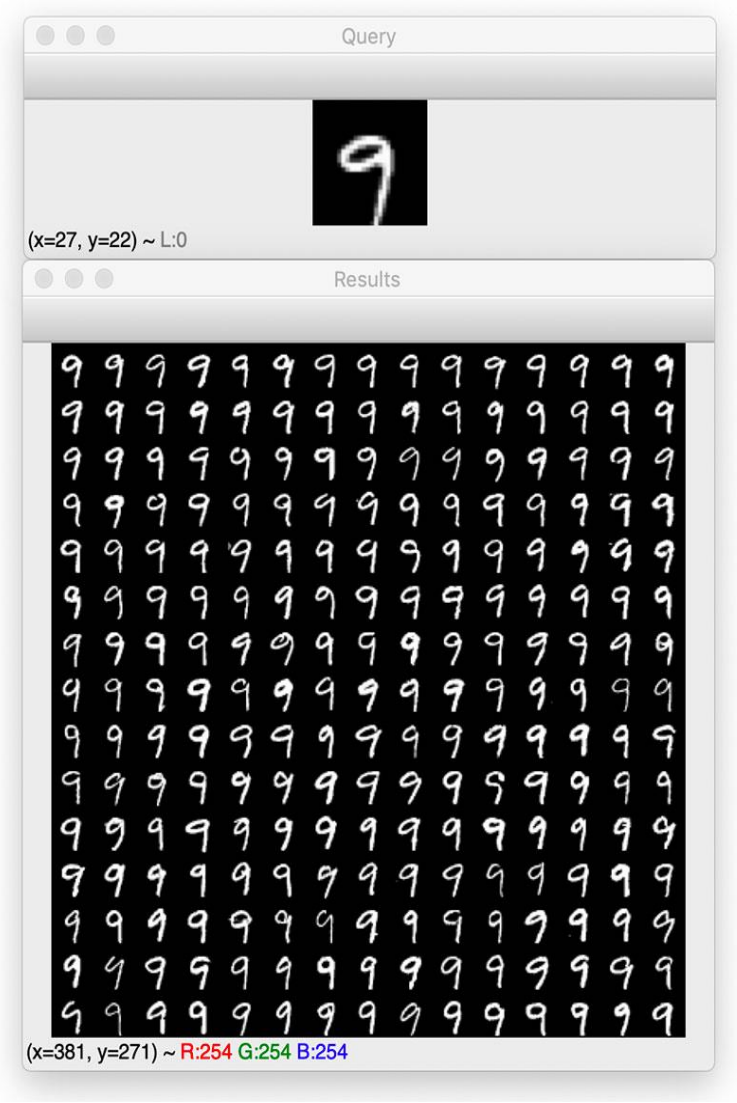

Fig 6:- MNIST query image.

\section{CONCLUSION}

As the technology is taking a rapid swipe over the smart world in developing and deploying the smart technologies we have to adopt and make use of it efficiently. We have developed model using the python libraries keras and tensor flow. Here we are training our data and then verifying it by providing proper inputs to which the output will be generated. The latent space as a feature vector for the images is calculated and the images are compared with each other and image retrieval techniques are used in various fileds of real world applications. Hence our model works effectively and efficiently.

\section{REFERENCES}

[1]. N. Hubens, "Build a simple Image Retrieval System with an Autoencoder", Medium, Aug. 2018.

[2]. M. I. Daoud, A. Saleh, I. Hababeh and R. Alazrai, "Content-based Image Retrieval for Breast Ultrasound Images using Convolutional Autoencoders: A Feasibility Study", 2019 3rd International Conference on Bio-engineering for Smart Technologies (BioSMART), pp. 1-4, Apr. 2019.

[3]. "Autoencoders for Content-based Image Retrieval with Keras and TensorFlow", PyImageSearch, Mar. 2020.

[4]. J. Brownlee, "Your First Deep Learning Project in Python with Keras Step-By-Sop", Machine Leaming Mastery, Jul. 2019.
[5]. "Different types of Autoencoders", OpenGenus IQ: Learn Computer Science, Jul. 2019.

[6]. I. A. Siradjuddin, W. A. Wardana and M. K Sophan, "Feature Extraction using Self-Supervised Convolutional Autoencoder for Content based Image Retrieval", 2019 3rd International Conference on Informatics and Computational Sciences (ICICoS), pp. 1-5, Oct. 2019.

[7]. M. A. Nielsen, Neural Networks and Deep Learning, [online] Available: http://neuralnetworksanddeeplearning.com.

[8]. A. Sze-To, H. R. Tizhoosh and A. K C. Wong, "Binary codes for tagging $\mathrm{X}$-ray images via deep de-noising autoencoders", 2016 International Joint Conference on Neural Networks (IJCNN), pp. 2864-2871, Jul. 2016.

[9]. Keras: the Python deep learning API, [online] Available: https://keras.io/

[10]. A. Baaj, "Keras Tutorial: Content Based Image Retrieval Using a Convolutional Denoising Autoencoder", Medium, Dec. 2019.

[11]. X. Yuan and C.-T. Li, "CBIR approach to building image retrieval based on invariant characteristics in Hough domain", 2008 IEEE International Conference on Acoustics Speech and Signal Processing, pp. 1209-1212, Mar. 2008.

[12]. K. Ramanjaneyulu, K. V. Swamy and C. S. Rao, "Novel CBIR System using CNN Architecture", 2018 3rd International Conference on Inventive Computation Technologies (ICICT), pp. 379-383, 2018. 